um die Grenzen der Meinungsfreiheit oder die Einschätzung der Gefahren von Drogen. Dahinter steht die Auseinandersetzung um die Macht über die Werteordnung des Grundgesetzes und die Beibehaltung der Gewaltenteilung, in der das Bundesverfassungsgericht Hüter, nicht Herrscher der Verfassung ist. « Richtig! Die Auseinandersetzung, an der sich das Allensbach-Institut mit seiner Umfrage - offenkundig mehr volens als nolens - beteiligt hat, geht um das Selbstverständnis einer pluralistischen Gesellschaft als politischer Gemeinschaft. Innerhalb einer Verfassungsordnung ist niemand /Herrscher « über die Verfassung, kein Einzelner und keine Gruppe oder Klasse, kein Verband und keine Partei, keine Elite und keine Institution - auch und schon gar nicht die vereinte Macht von Medien und Meinungsforschung!

\title{
Ulrich Mückenberger Flexibilisierung auf dem Gebiet des Familienrechts: die Mobil-Ehe ${ }^{\mathrm{I}}$
}

Die von Familienminister Blüm vorgelegten Reformvorschläge zur Mobil-Ehe stehen in Zusammenhang mit der jüngsten Veränderung des Personenstandsrechts. ${ }^{2}$ Im Kabinett war Einigkeit darüber erzielt worden, daß das Erfordernis zehnmonatigen Abstands zwischen letzter Scheidung und neuem Eheschluß, die überlangen Aufgebotsfristen wie auch das Kranzgeld schwerwiegende Freiheits- und Beziehungshindernisse darstellten, die zu beseitigen waren. Durch diese Flexibilisierungsansätze war Blüm dazu ermutigt worden, Veränderungsvorschläge, die er als Arbeitsminister erfolgreich betrieben hatte, nunmehr als Familienminister weiterzuverfolgen. Was er anstrebte, war eine Modernisierung des Beziehungslebens ohne Scheuklappen, eine Befreiung von bürokratischen Fesseln. Modern sind für den Minister die Selbständigkeit der Partner des Beziehungslebens und ihre Optionalität - nicht bevormundende persönliche Abhängigkeit. Jeder sei seines Glückes Schmied, betonte er unter Anspielung auf das in der Verfassung der Vereinigten Staaten verbriefte Recht nicht nur auf »life and liberty«, sondern auch auf »pursuit of happiness«.

Nicht nur die deutsche Ehe ist unproduktiv und langweilig geworden, in ganz Europa ist ein Rückgang erfinderischer Praktiken und der Beziehungsvielfalt eingetreten. Eine Sklerose ist zu verzeichnen, Sicherheit und Behäbigkeit lähmen Produktivität und Innovation. Nach Blüms Erkenntnissen muß die Ehe Verkrustungen abstreifen. Die Normal-Ehe ist tot - Mobil-Ehe ist angesagt. Gleit-, Schicht- und Wochenend-Ehe sind bereits verbreitet. Nach $\mathbb{1}$ Fertilitätsförderungsgesetz (FertFG) kann mittlerweile die Ehe - auch ohne sachlichen Grund - auf I 8 Monate befristet werden, im Falle von Neugründungen auf 24 Monate. Lieber befristet verheiratet als unbefristet ledig, pflegt der Minister zu sagen. Neugeschaffene Regelungen (wie die Beschleunigungsnovelle zum Zweiten Felizitätsfreisetzungsgesetz FelizBeschlG -) sehen die flexible Teilzeit-Ehe für Partner vor, die noch andere Verpflichtungen haben. Partner-Sharing hat bei Beziehungspionieren Freunde gefunden. Dazu tragen die Schutzvorschriften bei, die Mißbrauch verhindern: Ein

\footnotetext{
I Der vorliegende Kommentar bezieht wesentliche Denkanstoßße aus: Bundesministerıum fur Arbeit und Sozialordnung/Bundesanstalt fur Arbeit/Bundesministerium fur Familie, Senioren, Frauen und Jugend (Hrsg.), Mobil-Zeit. Ein Leitfaden fur Arbeitnehmer und Arbettgeber, Bonn 1995. 
Partner darf nicht mehr ohne weiteres zur Vertretung des anderen herangezogen werden; die Beziehung zum einen Partner darf nicht einfach deshalb aufgelöst werden, weil der andere Partner ausgeschieden ist usw.

Verbreitung gefunden haben Liebe-auf-Abruf und Potenzorientierte variable Liebeszeit (POZOVALZ) - beide Rechtsformen stellen in Westeuropa einzigartige Revolutionen auf dem Gebiet der Beziehungssouveränität dar. Das Partnerüberlassungsgesetz wurde liberalisiert: Nunmehr darf jeder Überlassungsfall bis zu neun Monate betragen, ohne daß sich der oder die Überlassene in Dauerehe einklagen kann. Wenn europaweit das staatliche Vermittlungsmonopol beseitigt sein wird, steht auch der kommerziellen Partnerüberlassung nichts mehr im Wege. Konsequenterweise will Minister Blüm mit seinem jüngsten Entwurf die Ketten-Ehe erleichtern.

Im Vorwort der Broschüre »Mobil-Ehe« schreibt Minister Blüm:

"Mobil-Ehe-was bedeutet das? Bisher haben wir immer von Teilzeit-Ehe gesprochen. Dieser Begriff ist aber zu eng und zudem mißverständlich. Teilzeit-Ehe hat - zu Unrecht - für viele einen negativen Klang. Teilzeit-Ehe gilt oft als nicht ganz vollwertige Ehe, weil sie vom ,Normalfall c der Voll-Ehe abweicht. Und wer will schon nur einen Teil und nicht ganze Arbeit leisten. Zudem wird vielfach unter Teil-Ehe nichts anderes als eine Halbtags-Ehe verstanden.

Aus diesen Denkschablonen mussen wir heraus. Wir müssen weg von starren, unflexiblen Eheformen. Wir brauchen Regelungen, die den Partnern mehr Freiraume geben, die Produktivität unserer Beziehungsarbeit verbessern und zusätzliche Bevölkerung schaffen.

Ein passender Begriff dafür ist Mobil-Ehe. Mobil-Ehe steht ebenso für eine nach individuellen Wünschen und Möglichkeiten vereinbarte Wochen- oder Tagesehezeit als auch für die flexible Jahresehe, gleitende Alters-Ruhezeit, angesparten Langzeiturlaub von der Ehe. Der Phantasie sind keine Grenzen gesetzt. Für viele, die Beziehung wollen, ermöglicht eine Mobil-Ehe unterhalb des Vollzeit-Standards überhaupt erst die gewünschte Beteiligung am Geschlechtsleben.«

Dieses Vorwort hatte Blüm r995 als Arbeitsminister geschrieben. ${ }^{3}$ Ministerin Nolte hatte damals weitblickend erklärt, die Umsetzung dieser Vorschläge »ist ein großer Gewinn für die Familien - und damit letztlich für die gesamte Gesellschaft «. ${ }^{4}$ Jetzt als Familienminister - brauchte Blüm diese Erkenntnisse nur den Verhältnissen anzupassen.

Hintergrund der Reform des Personenstandsrechts und der Mobil-Ehe sind tiefgreifende sozial-strukturelle Veränderungsprozesse. Das Erfordernis, beweglich zu sein, wechseln und lebenslang lernen zu können, macht auch vor dem Beziehungsalltag nicht halt. Daß sich die Wünsche nach Selbstverwirklichung in der Lebensgestaltung verstärkt haben, ist längst sozialwissenschaftliches Gemeingut.` Die Quoten von Scheidung, neuer Selbständigkeit, Wieder-Verheiratung haben sprunghaft zugenommen. Lebenslange Ehe, Normal-Ehe gehören der Vergangenheit an. Die Ketten-Ehe ist Realität. Nur hinkt das Gesetz wie so häufig nach.

Eine Tendenz zur »sequentiellen Polygamie - auch »serielle Monogamie « genannt wurde schon 1990 in einem Gutachten zur Zukunft der Familie hervorgehoben, das vom Bundeskanzleramt in Auftrag gegeben worden war. Damals hatte der Gutachter Franz-Xaver Kaufmann konstatiert: "Aus den Vereinigten Staaten wird über die Verbreitung eines Familienmusters >sukzessiver Ehen $<$ berichtet. ${ }^{6}$ Das Reformvorhaben von Minister Blüm zur Mobil- und Ketten-Ehe vollzieht diese Tendenz mit Mut und Augenmaß nach.

3 S. BMAS/BFA/BMFSFJ I99s (s. oben Fn. 1), S. 3.

4 S. BMAS/BIA/BMFSFJ 1995 (s, oben Fn. I), S. 5 .

s jurgen Hoffmann et al., Jenseits der Beschlußlage, Koln 1990, Kap. 1; s. auch Elisabeth Beck-Gernheim, Das normale Chaos der Liebe, Ffm. 1989.

6 Franz-Xaver Kaufmann, Zukunft der Familie. In: Perspektiven und Orıentierungen, Schriftenreıhe des Bundeskanzleramtes Band 10, Munchen: 1990, S. 100. 